\title{
Comparision of Preoperative Mri Findings with Intraoperative Findings in Patient of Fistula in ANO
}

\author{
Tanjum Kamboj ${ }^{1}$, Ram Kumar Verma ${ }^{2}$ \\ ${ }^{1}$ Post Graduate Resident, ${ }^{2}$ Professor \& Head, Department of Surgery, SGRRIM\&HS, Dehradun,UK
}

\begin{abstract}
MRI has become the method of choice for evaluating perianal fistulae due to its ability to display the anatomy of the sphincter muscles orthogonally, with good contrast resolution. In this article we compare MRI findings to intraoperative findings in patients of Fistula in Ano. This study is based on a retrospective analysis of 35 patients with a clinical diagnosis of perianal fistula
\end{abstract}

Keywords: Fistula in Ano, MRI, fistulogram,fistulectomy,seton.

\section{Introduction}

Fistula - in- Ano is an abnormal connection between the epithelialised surface of the anal canal or rectum and usually in continuity with one or more external openings in the perianal skin. Fistula - in- ano rarely heal spontaneously and requires surgical therapy to achieve a cure ${ }^{(1)}$

Traditionally been imaged by conventional fistulograms; the procedure involves cannulation of the external opening and injection of a water-soluble contrast into the fistula. This method has two main disadvantages: First, the primary tract and its extensions do not fill with contrast if they are plugged with pus or debris and, second, the sphincter muscle anatomy is not imaged and hence the relation between the tract, the internal/external sphincter, and the levator ani muscle is not revealed ${ }^{(2)}$.

A successful outcome after fistula surgery requires an accurate assessment of the fistula and patient expectations (especially in terms of risk to incontinence) (1).

\section{Corresponding author:}

\section{Dr Ram Kumar Verma,}

Professor \& Head, Department of Surgery, SGRRIM\&HS. Patel Nagar, Dehradun(248001). Uttarakhand, Email- drvermark2006@gmail.com
The improved surgical techniques have rendered steep fall in recurrence rate. With better training in colorectal surgery over recent decades and more experience in surgery of the anal sphincters, surgeons now have the confidence to try new methods for the treatment of an anal fistula to preserve the external sphincter ${ }^{(1)}$.

The external anal sphincter (a striated muscle) is clearly visualized on MRI. It is hypointense on T1W, $\mathrm{T} 2 \mathrm{~W}$, and fat-suppressed $\mathrm{T} 2 \mathrm{~W}$ images, and is bordered laterally by the fat in the ischioanal fossa.

The coronal images depict the levator ani muscle (levator plane), the identification of which is important to distinguish supralevator from infralevator infection ${ }^{(3)}$.

\section{Aims and Objectives}

- Preoperative mapping of fistula by MRI.

- To correlate the MRI finding with intraoperative finding

\section{Material and Method}

Study Design: A hospital based prospective type of study.

Study Period: The study was conducted over a period of 18 months from November 2017 to May 2019.

Study Area: The study was conducted in Shri Guru Ram Rai Institute of Medical \& health Sciences, 
Dehradun, India.

Study Unit: All patients with a diagnosis of Fistula in Ano

Sampling Technique: All cases of Fistula in Ano confirmed by clinical and radiological parameters were admitted in Department of Surgery.

Prior to initiation of study institutional ethical committee approval and informed consent from the patient/legal guardian after the full explanation of research protocol was taken.

Inclusion Criteria: The patients diagnosed as Fistula - in- Ano who will undergo surgical intervention during the study period.

\section{Exclusion Criteria:}

All congenital fistulas.

\section{Malignancy}

Inflammatory bowel disease patients

Incontinent patients

Patients with rectovaginal fistula

Cases unfit or refused for surgery.

\section{Study Planning:}

All the patients admitted were evaluated for fistula by history, clinical examination and investigation. Patients of Fistula in Ano were classified as anterior and posterior as per imaginary transverse line passing from the centre of anus, in lithotomy position. The position of external opening of fistula tract is described in o'clock position, where anterior midline position is taken as 12 o'clock and posterior midline is taken as 6 o'clock position.

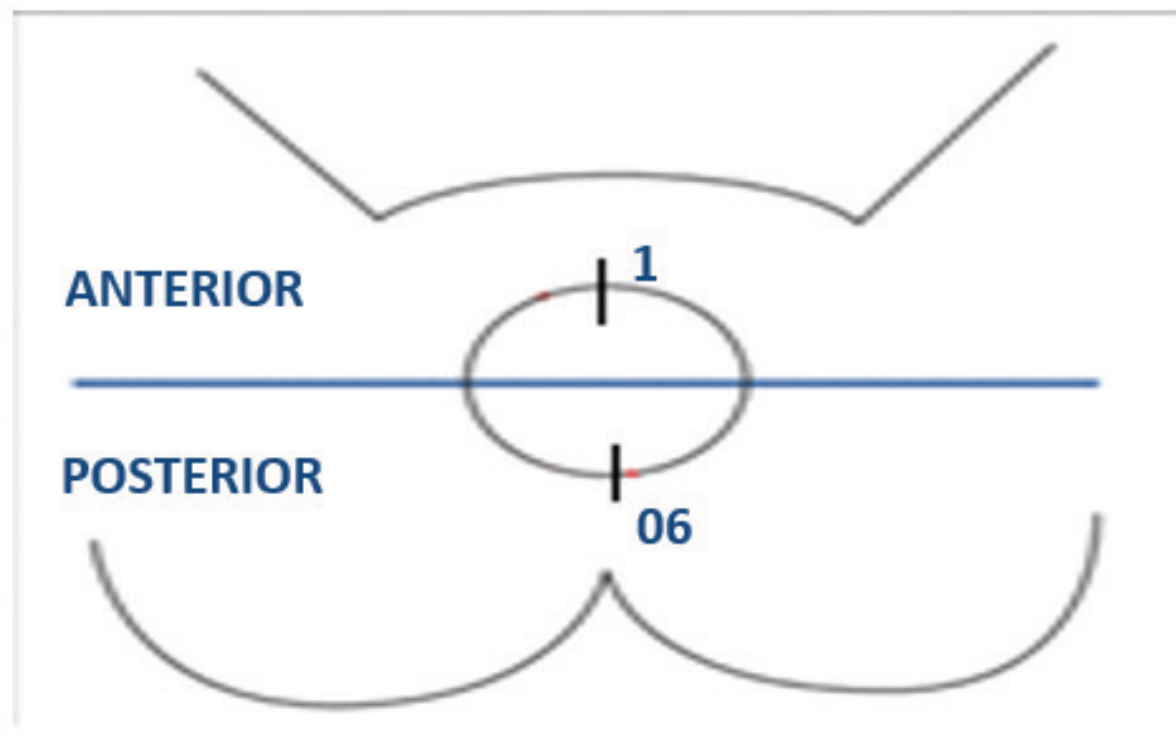

General/spinal anaesthesia was given to all patients. Perrectal examination was done after anesthesia and attempt was made to palpate the fistula tract. After palpation of fistula tract, a saline soaked gauge piece was inserted into the anal canal and methylene blue dye was injected from the external opening of the fistula tract. Staining of the gauge piece confirmed the communication of the fistula tract with the anal canal. Probing was done after dye injection, patients were then subjected to surgery (fistulectomy and /or seton placement). 
Findings

TABLE 1:PERIANAL EXAMINATION IN PATIENTS OF FISTULA IN ANO

\begin{tabular}{|c|c|c|c|c|}
\hline CASE NO. & $\begin{array}{l}\text { POSITION OF } \\
\text { EXTERNAL OPENING } \\
\text { OF FISTULA IN ANO }\end{array}$ & $\begin{array}{l}\text { NUMBER OF } \\
\text { EXTERNAL } \\
\text { OPENING }\end{array}$ & $\begin{array}{l}\text { DISTANCE OF } \\
\text { EXTERNAL OPENING } \\
\text { FROM ANAL VERGE }\end{array}$ & $\begin{array}{l}\text { POSITION OF } \\
\text { INTERNAL OPENING } \\
\text { OF FISTULA IN ANO }\end{array}$ \\
\hline 1. & $2 \mathrm{O}^{\prime} \mathrm{CLOCK}$ & 1 & $1.5 \mathrm{CM}$ & ABSENT \\
\hline 2. & 7 O'CLOCK & 1 & $3 \mathrm{CM}$ & ABSENT \\
\hline 3. & 1,3 O’CLOCK & 2 & $3 \mathrm{CM}$ & ABSENT \\
\hline 4. & $5 \mathrm{O}^{\prime} \mathrm{CLOCK}$ & 1 & $5 \mathrm{CM}$ & 10 O'CLOCK \\
\hline 5. & $9 \mathrm{O}^{\prime} \mathrm{CLOCK}$ & 1 & $2 \mathrm{CM}$ & ABSENT \\
\hline 6. & 2 O’CLOCK & 1 & $2 \mathrm{CM}$ & ABSENT \\
\hline 7. & 12 O’CLOCK & 1 & $4 \mathrm{CM}$ & 9 O’CLOCK \\
\hline 8. & 7,6 O’CLOCK & 2 & $2.5 \mathrm{CM}$ & ABSENT \\
\hline 9. & 3(Labia) & 1 & Labia & $12 \mathrm{O}^{\prime} \mathrm{CLOCK}$ \\
\hline 10. & 5 O’CLOCK & 1 & $2 \mathrm{CM}$ & 5 O’CLOCK \\
\hline 11. & $3 \mathrm{O}^{\prime} \mathrm{CLOCK}$ & 1 & $1.5 \mathrm{CM}$ & ABSENT \\
\hline 12. & $3 \mathrm{O}^{\prime} \mathrm{CLOCK}$ & 1 & $1 \mathrm{CM}$ & ABSENT \\
\hline 13. & $6 \mathrm{O}^{\prime} \mathrm{CLOCK}$ & 1 & $4 \mathrm{CM}$ & $6 \mathrm{O}^{\prime} \mathrm{CLOCK}$ \\
\hline 14. & 7 O'CLOCK & 1 & $4 \mathrm{CM}$ & ABSENT \\
\hline 15. & $5 \mathrm{O}^{\prime} \mathrm{CLOCK}$ & 1 & $2 \mathrm{CM}$ & ABSENT \\
\hline 16. & $5 \mathrm{O}^{\prime} \mathrm{CLOCK}$ & 1 & $1 \mathrm{CM}$ & ABSENT \\
\hline 17. & $1 \mathrm{O}^{\prime} \mathrm{CLOCK}$ & 1 & $2 \mathrm{CM}$ & ABSENT \\
\hline 18. & $1 \mathrm{O}^{\prime} \mathrm{CLOCK}$ & 1 & $1 \mathrm{CM}$ & ABSENT \\
\hline 19. & 10 O'CLOCK & 1 & $4 \mathrm{CM}$ & ABSENT \\
\hline 20. & 6 O'CLOCK & 1 & $0.5 \mathrm{CM}$ & $5 \mathrm{O}^{\prime} \mathrm{CLOCK}$ \\
\hline 21. & 9 O’CLOCK & 1 & $2 \mathrm{CM}$ & ABSENT \\
\hline
\end{tabular}


Cont... TABLE 1:PERIANAL EXAMINATION IN PATIENTS OF FISTULA IN ANO

\begin{tabular}{|c|c|c|c|c|}
\hline 22. & 11 O'CLOCK & 1 & $3 \mathrm{CM}$ & ABSENT \\
\hline 23. & 11 O'CLOCK & 1 & $3 \mathrm{CM}$ & $11 \mathrm{O}^{\prime} \mathrm{CLOCK}$ \\
\hline 24. & 9 O'CLOCK & 1 & $4 \mathrm{CM}$ & ABSENT \\
\hline 25. & $11 \mathrm{O}^{\prime} \mathrm{CLOCK}$ & 1 & $2 \mathrm{CM}$ & ABSENT \\
\hline 26. & 2 O'CLOCK & 1 & $2 \mathrm{CM}$ & $2 \mathrm{O}^{\prime} \mathrm{CLOCK}$ \\
\hline 27. & $2 \mathrm{O}^{\prime} \mathrm{CLOCK}$ & 1 & $3 \mathrm{CM}$ & ABSENT \\
\hline 28. & $2 \mathrm{O}^{\prime} \mathrm{CLOCK}$ & 1 & $2 \mathrm{CM}$ & ABSENT \\
\hline 29. & 6 O’CLOCK & 1 & $3 \mathrm{CM}$ & $6 \mathrm{O}^{\prime} \mathrm{CLOCK}$ \\
\hline 30. & 5 O'CLOCK & 1 & $4 \mathrm{CM}$ & ABSENT \\
\hline 31. & 3 O'CLOCK & 1 & $2 \mathrm{CM}$ & ABSENT \\
\hline 32. & 7 O'CLOCK & 1 & $2 \mathrm{CM}$ & ABSENT \\
\hline 33. & 11 O’CLOCK & 1 & $2 \mathrm{CM}$ & ABSENT \\
\hline 34. & $11 \mathrm{O}^{\prime} \mathrm{CLOCK}$ & 1 & $2 \mathrm{CM}$ & $11 \mathrm{O}^{\prime} \mathrm{CLOCK}$ \\
\hline 35. & 9 O’CLOCK & 1 & $3 \mathrm{CM}$ & ABSENT \\
\hline
\end{tabular}

TABLE2: POSITION OF EXTERNAL OPENING OF FISTULA TRACT(AS PER TRANSVERSE LINE)

Out of 35 patients admitted, position of external opening of fistula tract was anterior to transverse line in 23(65\%) patients. In $12(35 \%)$ patients position of external opening was posterior to transverse line.

\begin{tabular}{|l|c|c|}
\hline $\begin{array}{l}\text { POSITION OF EXTERNAL OPENING OF FISTULA } \\
\text { TRACT }\end{array}$ & NO. OF PATIENTS & PERCENTAGE \\
\hline ANTERIOR TO TRANSVERSE LINE & 23 & 65 \\
\hline POSTERIOR TO TRANSVERSE LINE & 12 & 35 \\
\hline TOTAL & 35 & 100 \\
\hline
\end{tabular}


TABLE 3: POSITION OF EXTERNAL OPENING OF FISTULA TRACT( DISTANCE FROM ANAL VERGE)

Out of 35 patients admitted, position of external opening of fistula tract within $3 \mathrm{~cm}$ distance from anal verge was seen in $27(77 \%)$ patients. In $08(23 \%)$ patients position of external opening was $>3 \mathrm{~cm}$ distance from anal verge.

\begin{tabular}{|l|c|c|}
\hline $\begin{array}{l}\text { POSITION OF EXTERNAL } \\
\text { OPENING OF FISTULA TRACT }\end{array}$ & NO. OF PATIENTS & PERCENTAGE \\
\hline$</=$ TO 3CM & 27 & 77 \\
\hline$>3 \mathrm{CM}$ & 8 & 23 \\
\hline TOTAL & 35 & 100 \\
\hline
\end{tabular}

\section{TABLE 4: NUMBER OF EXTERNAL OPENING OF FISTULA TRACT}

Out of 35 patients admitted, 33(94\%) patients had single external opening of fistula tract and 2(6\%) patients had multiple external openings of fistula tract.

\begin{tabular}{|l|l|l|}
\hline $\begin{array}{l}\text { NUMBER OF EXTERNAL OPENING } \\
\text { OF FISTULA TRACT }\end{array}$ & NO. OF PATIENTS & PERCENTAGE \\
\hline SINGLE & 33 & 94 \\
\hline MULTIPLE & 2 & 6 \\
\hline TOTAL & 35 & 100 \\
\hline
\end{tabular}

\section{TABLE 5: MRI FINDING CORRELATING WITH INTRAOPERATIVE FINDING}

Out of 35 patients, in 26 patients correlation was seen between MRI finding and Intraoperative finding in Fistula in Ano. No correlation was seen between the MRI finding and Intraoperative finding in 09 patient.

\begin{tabular}{|l|l|l|}
\hline & NO. OF PATIENTS & PERCENTAGE \\
\hline BOTH CORRELATE & 26 & 75 \\
\hline DO NOT CORRELATE & 09 & 25 \\
\hline TOTAL & 35 & 100 \\
\hline
\end{tabular}




\section{Discussion}

Total number of 35 patients of Fistula in Ano were admitted and studied. Patients who presented with clinical features of Fistula in Ano were included in the study. All patients of Fistula in Ano were admitted, clinical findings noted, all patients investigated with MRI pelvis diagnosis confirmed.

Out of 35 patients of Fistula in Ano in our study, in $27(77 \%)$ patients, the position of external opening of fistula tract was $<3 \mathrm{~cm}$ in distance from anal verge and in $8(23 \%)$ patients, the position of external opening of fistula tract was $>3 \mathrm{~cm}$ in distance from the anal verge. In a study done by Alexander et. al, in $92.5 \%$ patients of Fistula in Ano, the position of external opening of fistula tract was $<3 \mathrm{~cm}$ in distance from anal verge and in $11 \%$ patients, the position of external opening of fistula tract was $>3 \mathrm{~cm}$ in distance from the anal verge ${ }^{(4)}$.

the patients of Fistula in Ano were treated with fistulectomy, intraoperative findings were correlated with MRI findings. In 26(75\%) patients, intraoperative findings were similar to MRI findings and in 09(25\%) patients, intraoperative findings were different from MRI findings. In our study of patients of Fistula in Ano, correlation between the MRI and intraoperative findings was seen in $75 \%$ patients. As per study by Jajoo et al, 56 patients out of sixty completely correlated with MRI for primary tract of Fistula in $\mathrm{Ano}^{(5)}$. In a study done by Ali $\mathrm{K}$ et al and Mehmet R.O. et al, MRI finding was concordant with operative finding in $83.1 \%$ patients of Fistula in $\mathrm{Ano}^{(6)}$.

Out of 09 patients of fistula in ano whose MRI and intraoperative findings did not match, 07(78\%) patients were having anterior fistula in ano and $02(22 \%)$ patients were having posterior fistula in ano.

\section{Conclusion}

Anterior fistula in ano are more common than posterior fistula. External opening of fistula tract was anterior to transverse line in $65 \%$ and posterior to transverse line in $35 \%$ patients of Fistula in Ano

In $75 \%$ cases of Fistula in Ano correlation was seen between the MRI finding and intraoperative finding, but no correlation was seen between the MRI finding and intraoperative finding in 25\% patients of Fistula in Ano.

Out of 35 patients of Fistula in ano in the study 23 patients had anterior fistula, in 07 patients MRI findings did not correlate with intraoperative findings.

Out of 35 patients of Fistula in Ano in the study 12 patients had posterior fistula, in 02 patients MRI findings did not correlate with intraoperative findings.

In $25 \%$ patients of Fistula in Ano in which no correlation was seen between the MRI finding and intraoperative finding, $78 \%$ patients in this group were having anterior fistula and $22 \%$ patients were having posterior fistula.

Therefore it is observed that anterior Fistula in Ano are less accurately mapped on MRI.

To summarise, evaluation of an Fistula in Ano by MRI, provides most of the details necessary for accurate evaluation.

Funding: No Funding Sources

Conflict of Interest : None declared

Ethical approval: The study was approved by the institutional ethical committee.

\section{References}

1. Norman S. Williams, Chrstophe r JK, Bulstrods

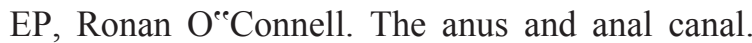
Chapter - 69, In: Bailey and Love ${ }^{\text {ee }}$ s short practice of surgery. 25th Edn. London : Hodder Arnold; 2008. p.1262- 1264.

2. Halligan Steve, Jaap Stoker. Imaging of fistula in ano. Radiology. 2006;239:18-33. [PubMed]

3. Stoker J, Fa VE, Eijkemans MJ, Schouten WR, Laméris JS. Endoanal MRI of perianal fistulas: The optimal imaging planes. Eur Radiol. 1998;8:12126. [PubMed]

4. Gunawardhana PA, Deen KI. Comparison of hydrogen peroxide instillation with Goodsall's rule for fistula-in-ano. ANZ J Surg 2001;71:472-4.

5. Beets-Tan RG,Beets GL, van der Hoop AG, Kessels AG, Vliegen RF, Baeten CG, van Engelshoven 
JM. Preoperative MR imaging of anal fistulas: does it really help the surgeon?. Radiology.2001 Jan;218(1):75-84
6. Alexander N, Sanniyasi S, Joseph J, Paramasivam S. A Single Center Study of 80 Cases of Fistula-inano. Int J Sci Stud 2016;4(8):122-125. 